\title{
Fallopian Tube Mucinous Metaplasia
}

National Cancer Institute

\section{Source}

National Cancer Institute. Fallopian Tube Mucinous Metaplasia. NCI Thesaurus. Code C126463.

Metaplastic mucinous changes in the fallopian tube epithelium. It may be seen in association with gynecological, appendiceal, or colonic mucinous tumors, or chronic inflammation. 